\title{
VIGENCIA DEL ESQUEMA CONDUCTUALISTA EN LOS ESTUDIOS INTERNACIONALES: SU VIABILIDAD EN AMÉRICA LATINA
}

\author{
Roberto Durán
}

Si bien el conductualismo se ha incorporado recientemente a los estudios de las relaciones internacionales de América Latina, no se puede desconocer su aporte en cuanto a que ha implicado un reenfoque de la:investigación y a su acento empiricista. Para comprobar lo anterior, el autor se refiere primeramente a la validez metodológica del esquema conductualista y su influencia en las ciencias sociales en general. Más adelante, explica la vigencia de esta corriente en los procesos de cooperación y confrontación en la región latinoamericana y sugiere la elaboración de un modelo para diagnosticar conflictos y evaluar soluciones factibles, planteando hipótesis de trabajo de acuerdo a tres escenarios posibles.

Fundamentar la vigencia u obsolescencia de un esquema teórico es tema y labor permanente en toda disciplina que se pretenda definir como científica. En cierto sentido, la elaboración, comprobación, refutación y posterior reelaboración de los argumentos conceptuales y metodológicos que postula un determinado esquema constituyen el núcleo central de toda teorización, en especial si ésta tiene por objetivo mejorar el rigor de una disciplina relativamente nueva como son los estudios internacionales.

Como es sabido, el esbozo de las primeras teorías de relaciones internacionales se puede encontrar en algunos estudios jurídicos y politológicos de hace unos cincuenta años. En la medida en que los estudios internacionales empiezan, por un lado, a diferenciarse de una óptica puramente jurídica y, por otro, a adquirir un perfil propio en la ciencia política, también se inicia el periplo teórico que hoy en día constituye una de las literaturas más extensas de las ciencias sociales.

Ciertamente, la maduración conceptual de esta disciplina está en desarrollo y se observan situaciones contradictorias. Por lo pronto, este desarrollo no es similar en todos los casos y contextos. Hay 
teorías cuyo nivel de abstracción no facilita la contrastación empírica y otras que han caído en un empiricismo craso. Existen centros académicos cuya labor docente y de investigación se define alrededor de una determinada escuela o corriente de pensamiento, otros en los que predomina una peculiar concepción de pragmatismo académico y otros en los que circunstancias extraacadémicas no facilitan teorización alguna.

Ahora bien, el conductualismo (behaviorism) ha sido una escuela influyente en las ciencias sociales desde los inicios de los años cincuenta. De sus postulados no se desprende un marco teórico excesivamente abstracto, aunque muchos de sus conceptos tienen implicaciones claramente generalizantes y generalizables. Durante un tiempo, el conductualismo asumió algunas características hegemónicas en las ciencias sociales, particularmente en la sociología y luego en la ciencia política anglosajona. Dicha situación derivó de la enorme influencia de investigaciones sociológicas centradas en grupos primarios y comunidades, fenómeno social y político cuya relevancia se evidencia hasta mediados de los años sesenta. De ahí deriva también la connotación empiricista que suele imputarse al conductualismo.

En una primera fase, el conductualismo otorga mayor rigor a las. investigaciones y estudios decididamente empiricistas de la sociología y la ciencia política norteamericanas de hace treinta años. En la medida en que los estudios internacionales tenían un status discreto en el ámbito de la ciencia política de entonces, el peso de esta escuela es igualmente discreto, al menos en un principio. En una segunda fase, corrientes opuestas al conductualismo restauran la preeminencia de esquemas conceptuales inspirados en esquemas organicistas, en un complejo proceso que entremezcla posturas antiempíricas con tendencias propositivistas. Paralelamente, el status académico de los estudios internacionales tiende a consolidarse en ese período (mediados de los sesenta), y en cierto modo el debate entre "utopistas" y "realistas" no sólo tiene una raíz eminentemente conceptual, sino además una postura metodológica para observar y evaluar el sistema político internacional de fines de los cincuenta y durante toda la década de los años sesenta.

Cabe señalar que la inserción del conductualismo en los estudios internacionales tiene menos implicaciones hegemónicas que en el caso de otras disciplinas. Por lo pronto, varios teóricos realistas instrumentalizan muchas de sus premisas para contrastar -exitosa- 
mente o no-sus hipótesis. A la inversa, varios conductualistas suelen valerse del realismo para otorgar a sus proposiciones una visión de conjunto, la que no siempre se desprende del análisis de casos dispersos y aislados. Esta mutua instrumentalización se ha extendido a otros esquemas, por lo que es usual encontrar una vasta literatura acerca de organizaciones internacionales, procesos integracionistas, enfoques político-estratégicos y otros que incluyen conceptos y procedimientos metodológicos propios del conductualismo. Tal ha sido la trayectoria de esta corriente en los últimos veinte años.

$\mathrm{El}$ abundante bagaje de estudios y literatura que hace uso del esquema conductualista ha hecho suponer la inexistencia de esta escuela como teoría en sí. Sin desconocer la validez y pertinencia de sus conceptos, son sus procedimientos, implicaciones y conclusiones metodológicas los más meritorios. Es decir, las proposiciones del conductualismo aportan más por la forma en que éste se aproxima al análisis de los hechos que por la vía de la abstracción conceptual.

A este respecto, nos permitiremos algunas puntuaciones. Primero, toda teoría es un conjunto de conceptos susceptibles de ser corroborados o refutados por la realidad, entendiendo que ésta última no se restringe a lo meramente cotidiano sino a una acepción más amplia, que incluye una perspectiva histórica y estructural. Segundo, a fin de comprobar la consistencia de sus conceptos y definiciones, así como la coherencia de éstos con la realidad, se hace uso de técnicas e instrumentos metodológicos atingentes, adaptados a los requerimientos teóricos de lo que se quiere estudiar o investigar. Al igual que el resto de las ciencias sociales y de toda disciplina científica en general, la teoría y su metodología constituyen una misma entidad. Las extrapolaciones conceptuales y el uso magnificado de instrumentos metodológicos, acarrea a menudo interpretaciones livianas y confusas sobre el quehacer de algunas teorías. En el caso del conductualismo, la extrapolación acrítica de muchos de sus conceptos se hace para paliar la pobreza conceptual de ciertos esquemas, en especial cuando sus proposiciones deben ser empíricamente contrastadas. Otras veces, lo que se extrapola es la forma de aproximarse al objeto de estudio, la manera de definir el problema a investigar y el modo de construir variables, indicadores e índices. Aquí no se trata de hacer uso de las reglas básicas y universales de la metodología científica, sino de utilizarlas tal cual to hace el conductualismo. Paradojalmente, es en estos casos en los que sus detractores abundan, 
restringiendo unilateralmente su quehacer teórico a una mera "utilidad en lo metodológico".

Sin embargo, el cúmulo cuantitativo y cualitativo de investigaciones y estudios inspirados en los postulados y proposiciones de esta corriente da cuenta de un cuadro bastante diferente. Conceptos tales como "actor", "acción racional" y la trilogía "percepción-estímulo-motivación" han tenido y tienen un uso frecuente. Son también los conceptos más extrapolados y, por lo mismo, muy sensibles a interpretaciones vagas y ambiguas.

En el caso de la "acción racional", parte no despreciable de autores lo interpreta despojado de su doble connotación weberiana, asimilando su contenido a una simple oposición entre lo racional vs. lo irracional. Esto es particularmente notorio al examinar la política exterior de las pequeñas y medianas potencias respecto de las superpotencias o la política seguida por los Estados pequeños (Weak States, Isolated States) entre ellos y respecto de países de mayor envergadura. En estos casos, la racionalidad esperada de una política externa rara vez es coincidente con su racionalidad real, habida cuenta que las condiciones en las que se desenvuelve dicha política están permanentemente constreñidas por escasez de recursos, alteraciones inesperadas de procesos regionales y subregionales y, lo que es casi una norma, debido a los bruscos cambios que operan en los sistemas políticos de dichos Estados. Es el comportamiento errático y asistemático de estas políticas lo que suele confundirse con conductas irracionales, en circunstancias que la irracionalidad más tiene que ver con errores de diagnóstico o con una ineficiente asignación y/o implementación de recursos. También existe una inveterada tendencia a acomodar los hechos a un esquema previo, forzando hasta el extremo la información disponible. Son las tradicionales acepciones de una política externa pragmática, que se asimila a un modelo racional con arreglo a fines o una política exterior dogmática o "principista", la cual se asimila a un modelo racional de acuerdo a valores. Como consta tradicionalmente, la real-politik es una entremezcla de principios y pragmatismo, en la que el predominio de una u otra obedece a factores circunstanciales, tanto en el ámbito de la política interna como en el de las relaciones interestatales. Las políticas exteriores latinoamericanas en su conjunto, a nivel de los vínculos bilaterales y en el plano de las negociaciones multilaterales también han fehacientemente demostrado esta dualidad. Ha habido 
períodos, por ejemplo, en que una determinada consistencia entre medios y objetivos constituye un punto de referencia importante en la tradición diplomática de un país y, cuando así ocurre, đicha consistencia guarda una estrecha relación con la estabilidad política interna del mismo país. De otra forma, ¿cómo podrían entenderse alteraciones sustanciales entre las políticas exteriores de países latinoamericanos de una década à otra, los profundos cambios en sus estilos diplomáticos y la subsecuente crisis que estos cambios han provocado en las relaciones intrarregionales? En última instancia, los cambios en los estilos bilateral o multilateral proyectan alteraciones previamente manifestadas en la voluntad política de los Estados, la cual es voluble a transformaciones que operan al interior de cada sistema político. En suma, la permanencia de ciertos principios y formas de comportamiento en política exterior está supeditada a la capacidad con la que dichas políticas se adaptan a la evolución y transformaciones de la política mundial o regional, adaptación que implica, por un lado, acomodar sus objetivos a recursos escasos y, por otro, adecuarlas al status que les confiere el errático sistema internacional. Es en este contexto en el cual cabe catalogar el grado de racionalidad o irracionalidad en el que incurre el comportamiento de una política exterior.

Un uso igualmente ambiguo se ha hecho y se hace de la relación entre "percepción-estímulo-motivación", la cual también ha servido para describir y explicar el comportamiento y evolución de las polít:cas externas de los Estados. Sin embargo, lo normal es encontar un uso parcial de tal esquema, por cuanto la literatura al respecto abunda en ejemplos de percepción y escasea notablemente en el uso de los conceptos de estímulo o motivación. Es posible que ello se deba a la importancia que, en general, se atribuye en los estudios de imagen internacional a los mecanismos de percepción, tema que ha ocupado el tiempo de muchos teóricos, pero que sobre todo ha sido una preocupación permanente de los policy-makers. Ingentes recursos de todo orden han sido destinados para acrecentar y mejorar los análisis de percepción internacional y regional, recursos que innecesariamente han amplificado el área de influencia de la burocracia estatal en detrimento de una asignación más equitativa, por ejemplo, respecto de centros académicos. De todas formas, el solo hecho de procurar un mejoramiento en la capacidad de elaborar diagnósticos por parte de la burocracia es un estímulo para la profesionalización de los 
servicios exteriores latinoamericanos, aunque sólo se trate de un esfuerzo ocasional. Por otro lado, el apasionado debate que hace años dividió a psicólogos y sociólogos respecto de las diferencia entre estímulo y motivación tiene menor perfil en su aplicación al ámbito de los procesos internacionales. Quizás ello se deba a que el propio concepto de acción racional en lo internacional no tiene la diversificación que tiene en el plano de las relaciones interpersonales o en el de la estructura social y cultural de una sociedad. Por cierto, la extrapolación conceptual sólo se refiere a los aspectos más esenciales del concepto del que se quiere hacer uso, por lo que distinguir entre motivación o estímulo en política internacional es irrelevante. No obstante, la literatura sobre el tema confunde frecuentemente los objetivos de una política externa con las motivaciones que están detrás de ciertas posturas coyunturales. Si los objetivos están condicionados por factores y situaciones de tipo estructural, muchas veces dificilmente manejables, las posiciones bilaterales o multilaterales que circunstancialmente adopta una política obedece a situaciones igualmente circuntanciales, que van desde hechos inesperados y decisivos que marcan un período de la política mundial o regional hasta la predisposición cotidiana de quienes toman las decisiones más fundamentales de la política exterior del Estado. Este es un tema particularmente ilustrativo en las políticas externas de algunos países de América Latina, sobre todo en aquéllos en los que la burocracia pública no está dotada de un servicio exterior profesional y despersonalizado.

Recapitulando lo adelantado en puntos anteriores, hacia fines de los años sesenta y durante la mayor parte de la década de los setenta, el conductualismo cedió ante otras escuelas, precisamente en el período en que los estudios internacionales consolidan un status académico definitivo en el ámbito de la ciencia política y en general entre las ciencias sociales. La preeminencia de otras escuelas irrumpe en un momento particularmente débil para el conductualismo, toda vez que esta corriente está en el centro de un álgido debate que coincide con una profunda crisis de identidad en la que se sume la sociología y la psicología social anglosajona y europea-occidental. Son los efectos de esa crisis lo que desalienta a muchos seguidores de esta escuela, situación que se prolongó varios años.

Paralelamente, durante esos años se introducen los estudios internacionales en América Latina, labor hecha con el auxilio de las 
escuelas y corrientes entonces predominantes. En la medida en que la disciplina se introduce a manera de especialidad o deriva de otras con mayor tradición académicá (derecho, historia y ciencias económicas), el conductualismo no hace su aparición sino muy recientemente.

Como es usual en medios académicos latinoamericanos, su llegada la hacen quienes se han especializado en universidades anglosajonas o -en menor grado- europeo-occidentales. Otras veces proviene de sociólogos, psicólogos o psiquiatras, casos en los que el conductualismo no sólo les resulta un esquema familiar, sino además es la forma de aproximarse a un nuevo objeto de estudio.

En cualquier forma, es obvio que el conductualismo no puede reclamar en los estudios internacionales de América Latina una tradición como la adquirida por el realismo, el integracionismo, los esquemas desarrollistas, la dependencia y otras corrientes. Del mismo modo, su influencia, por ejemplo, en la sociología latinoamericana fue y continúa siendo muy parcial, y aparte de las experiencias acumuladas en estudios de comportamiento y tendencias electorales (labor en la que hoy día están empeñados más politólogos que sociólogos) o en análisis de opinión pública (también muy disputados por politólogos y psicólogos-sociales), la presencia real de esta corriente es muy exigüa. Esto explica las características básicamente exploratorias a las que debe ceñirse una investigación o estudio de corte conductualista actualmente, especialmente en centros latinoamericanos. Hay que tener presente que el diseño y puesta en marcha de este tipo de investigaciones exige recursos financieros y humanos cuantiosos, exigencia que se ha agudizado con la introducción de modelos estadísticos y computacionales. Al mismo tiempo, la implementación de estos estudios produce sentimientos encontrados y oscurantistas en algunos centros académicos de la región, ya sea porque se les entiende como la punta de lanza de un "malicioso neo-positivismo" o porque se les atribuye una ineficiente duplicidad de esfuerzos (respecto de los que se llevan a cabo en otros centros, principalmente anglosajones). Sería indebido negar la orientación netamente empírica de este esquema y, por lo mismo, sería incorrecto negarle una raigambre positivista. Pero tampoco cabe descalificarlo por esa razón, sobre todo si se hace estrictamente a priori, desconociendo su reciente incorporación en las ciencias sociales latinoamericanas y prejuzgando sobre resultados que aún están por verse. 
La pertinencia de un enfoque conductualista en las relaciones interregionales e internacionales de los países latinoamericanos puede argumentarse de la siguiente manera. En la dinámica política de los procesos interregionales se pueden observar comportamientos que no pueden subsumirse a la dinámica del intercambio comercial ni tampoco son consecuencia de recurrentes vínculos diplomático-formales. Por ejemplo, las tensiones provocadas por centenarios diferendos limítrofes no son sólo explicables por el mayor o menor apego que muestren los gobiernos hacia el derecho internacional o por el grado de autoritarismo o democratización de determinados sistemas políticos. Sin excluir su valor como variables intervinientes, el hecho es que en el origen y desarrollo de procesos bilaterales como el mencionado y otros, hay fenómenos de comportamiento cuyo radio de acción es autómono de instancias formales y en los que se entremezclan elementos de diversa índole (socialización, intereses contrapuestos, liderazgos efímeros, nacionalismos tradicionales y exacerbados, etc.). Si bien dicha autonomía es relativa y condicionada a factores momentáneos, estos fenómenos conductuales están presentes en el contenido de las políticas exteriores, ya sea por la vía de expresiones manifiestas o bajo formas latentes. Si así no fuera, no serían un aspecto esencial del discurso oficial de ciertos Estados ni tampoco parte integral de la entelequia de sus políticas exteriores.

A nuestro juicio, las motivaciones que inducen a posturas agresivas y/o cooperacionistas en la política bilateral latinoamericana son el producto de comportamientos inducidos por una pluralidad de indicadores, entre los cuales destacan tres.

En primer lugar, al no haber una neta difrenciación entre las políticas públicas que emanan de un aparato estatal autónomo y las que provienen de ámbitos extrasistémicos (poder oligárquico y/o personalmente concentrado), la política exterior de algunos países queda expuesta a distorsiones ideológicas extremadamente cambiantes. Necesariamente, esto repercute en orientaciones asistemáticas y escasamente constantes, lo que a la postre redunda en posturas crecientemente atadas a circunstancias o coyunturas. Al haber una exagerada confusión entre intereses nacionales e intereses particulares, las políticas externas de esos regímenes desdibujan el sentido y la vigencia de los intereses permanentes del Estado, lo cual, en el mediano y largo plazo, les ha significado una ostensible pérdida de credibilidad en el sistema internacional. Cabe señalar que éste es un proceso reciente, por cuanto hubo casos en que regímenes uniperso- 
nales latinoamericanos acrecentaron la influencia de sus respectivos países, $y$, por ende, tuvieron gran credibilidad internacional. La diferencia entre esos años (fines de la década de los cuarenta y parte importante de la de los cincuenta) y los de ahora radica en el grado de permeabilidad e interpenetración de los sistemas políticos. Mientras duró la Guerra Fría y el período de reconstrucción de post-guerra, la influencia de éstas estaba mediatizada por un sistema internacional polarizado y en plena recomposición. Con la llegada de la distensión, de la mundialización del intercambio comercial y científico-tecnológico y la emergencia de nuevos polos de poder e influencia, el sistema internacional entró en una fase de creciente transnacionalización, proceso al cual se incorporó un número apreciable de nuevos Estados y en el que varios países latinoamericanos asumieron un rol importante. Las principales líneas de ese proceso han seguido evolucionando y no obstante las características recesivas del desarrollo político latinoamericano en los años setenta y sus efectos en los niveles de las relaciones interregionales y en los vínculos de la región con el resto del mundo, el contexto global en el cual se diseñan e implementan las políticas externas de estos países se ha ampliado notablemente $y$, por lo mismo, estas mismas políticas son mucho más sensibles a los requerimientos y presiones del entorno internacional.

En segundo lugar, las relaciones bilaterales y multilaterales de los países latinoamericanos entre sí y de éstos con otros Estados y/o bloques de países, están condicionadas al desarrollo y mejoramiento de servicios exteriores cuya profesionalización y funcionalidad no es siempre uniforme. Con la estricta salvedad de algunos casos, la mayoría de los países latinoamericanos no han estructurado servicios diplomáticos cuya eficiencia se mida en función de resultados concretos, los que a su vez permitan dar cuenta de la proyección internacional alcañzada por sus respectivos países en los ámbitos bilateral o multilateral, sea esto en el corto o mediano plazo. La instrumentalización de la diplomacia como expresión externa de una ideología gobernante o su mera utilización como mecanismo de recompensa y/o prebendas es una constante en la región, situación que se agudiza o se suaviza para cada caso, pero que rara vez pierde esa forma. Se podría argumentar -y con mucho fundamento- que ésta es una variante de la falta de autonomía de la burocracia pública latinoamericana, como se señalara previamente. Pero a esto hay que añadir la errada percepción que estos países tienen del sistema político inter- 
nacional, formulada mediante diagnósticos incompletos y transmitida a los distintos niveles decisionales por una burocracia ineficiente y mal dotada. En cierta forma, esta inadecuación explica los altibajos y abruptos cambios que se observan, por ejemplo, en las posturas multilaterales de ciertos gobiernos, sean éstos en el terreno de negociaciones regionales o extrarregionales. Así también se explica la personalización que han adquirido determinados víaculos bilaterales, casos plenamente confiados al tacto, buen manejo y pericia de embajadores o funcionarios de confianza, en vez de confiarlos a los canales burocráticos formales. Un primer resultado de todo esto es la descalificación de los propios servicios exteriores, órganos competentes en la ley e incompetentes en la realidad. Un segundo resultado es la descoordinación en la que cae una política exterior insuficiente y deficientemente informada, con todos los efectos que involucra en la presencia, status y rol internacional que debe asumir el país.

El tercer grupo de indicadores que inciden (positiva o negativamente) en la adopción de políticas agresivas o conciliadoras entre los países latinoamericanos se refiere específicamente al ámbito multilateral. Normalmente, se suele asimilar la concepción de cooperación a la de conciliación, entendiendo que esta última es producto de la primera y/o viceversa. Muy ocasionalmente se tiene en cuenta que los mecanismos de conciliación no necesariamente involucran la existencia de mecanismos de cooperación y muchos estudios no auscultan suficientemente los paréntesis conflictivos en los que caen algunos circuitos de cooperación. Lo que sucede es que se trata de dos actitudes diferentes, ya que mientras la conciliación es una predisposición compartida por dos o más países para dirimir una crisis de intereses, la cooperación es una instancia para aunar intereses. Los hitos conciliatorios que han caracterizado a las relaciones bilaterales -y a veces a las multilaterales-de América Latina han marcado etapas importantes en la cooperación regional, pero no necesariamente ha existido un vínculo dependiente entre uno y otro. Ciertamente, han habido conflictos bilaterales cuyas implicaciones han derivado en crisis graves e incluso insalvables para con determinados mecanismos de cooperación, pero no se ha dado el caso a la inversa. Ahora bien, ¿en qué punto se toca la conciliación bilateral con la concertación multilateral? Por lo pronto, son negociaciones diferentes, en su origen y estilo. Mientras la conciliación de intereses dirime sobre posturas contrapuestas en asuntos o temas muy específicos y 
las negociaciones diplomáticas se ajustan a una agenda de trabajo muy acotada, la conjunción de intereses se refiere a asuntos o temas de mayor amplitud y las negociaciones comprenden una agenda más extensa. El esquema tridimensional percepción-estímulo-motivación también asume un papel diferente en uno y otro caso. En la conciliación, el estímulo y retroalimentación de actitudes está limitado a los comportamientos de muy pocos actores, exactamente lo contrario de lo que sucede en la cooperación. En segundo lugar, la disposición para negociar diplomáticamente la solución de una crisis de intereses es distinta de la que intenta concertar y articular posturas respecto de terceros actores. En el primer caso suelen estar de por medio ciertos intereses permanentes de los Estados, mientras que en el segundo juega un rol más preminente el manejo de factores diplomáticos, comerciales, políticos u otros. En otro sentido, son racionalidades político-diplomáticas diferentes; en la conciliación la balanza se inclina por los fines y en menor grado -instrumentalmente- por lo valores, y normalmente ocurre lo contrario en la cooperación.

La crisis enfrentada por las instituciones y mecanismos de cooperación latinoamericanos desde mediados de los años setenta en adelante, es un caso en el que conciliación y concertación de intereses se entrecruzaron conflictivamente. La proliferación del autoritarismo durante la década pasada redefinió el carácter de las relaciones intrarregionales y, por esa vía, también se redefinió el alcance de la cooperación regional. El énfasis en orientaciones nacionalistas estimuló la implementación de políticas exteriores apegadas a criterios político-estratégicos y en ese sentido el multilateralismo latinoamericano se limitó extremadamente. Paralelamente, la percepción de una deslegitimación creciente del multilateralismo mundial y el letargo político de la integración regional profundizaron la crisis del multilateralismo latinoamericano, la que hacia fines de los años setenta se amplía a niveles institucionales.

Ahora bien, si bien los multilateralismos mundial y regional se insertan en contextos diferentes, las características de la crisis institucional de ambos tienden a asemejarse, al menos en un primer momento. Primero, durante los años en que proliferan las organizaciones intergubernamentales, su rol político quedó sujeto a diversas interpretaciones. Por ejemplo, el activo papel asumido por las Naciones Unidas en la solución de conflictos regionales o en las crisis que amenazaban la paz mundial en los años cincuenta y primer tercio de los sesenta, no fue seguido en períodos posteriores. Más aún, entre 
mediados de los sesenta y los primeros años post-crisis energética $1973 / 74$, las relaciones interestatales directas vuelven a retomar la iniciativa en el resguardo de la paz y seguridad mundiales, es desmedro del rol político que al respecto habían asumido años antes las Naciones Unidas. Las recesiones económicas de mediados de los setenta y principios de los ochenta y sus posteriores rezagos, así como los acuerdos y tratados directamente celebrados entre países en materias de intercambio comercial y cultural, diferendos políticos, limítrofes y de cualquier otro orden, continúan restringiendo el radio de influencia del multilateralismo mundial. En otro contexto geográfico, la crisis de algunos órganos del sistema interamericano también ha implicado una aguda polémica acerca del papel político efectivo que le cabe a este sistema, por ejemplo, en la solución de diferendos $o$ en asuntos que afectan a la seguridad hemisférica.

La pregunta es, ¿en qué términos o a qué nivel se entendía y se entiende hoy la concepción de "actor internacional" que tan frecuentemente se atribuye a los organismos intergubernamentales? Si proliferan acepciones encontradas respecto de los atributos políticos de los actores internacionales, las hay aún más acerca de las condiciones que reúnen los organismos intergubernametales para acreditar su condición de tales. Este es un problema relacionado con los indicadores mediante los cuales se percibe el rol político de estas instancias. Para una política exterior orientada hacia lo multilateral, su percepción está dirigida a estimular mecanismos y procedimientos político-diplomáticos que aseguren una presencia activa y permanente de cada país en esas instancias. En el caso opuesto, lo multilateral se percibe con bastante incertidumbre, lo cual estimula una conducta renuente a la concertación. Ambos casos se han dado en los planos mundial y regional, lo que nos lleva a afirmar que el reforzamiento o debilitamiento de la legitimidad del multilateralismo pasa por la percepción que se tiene de sus logros y fracasos. Así, al mismo tiempo que dicha percepción permite una visión de conjunto sobre los efectos de determinados procesos multilaterales, ésta misma evalúa las posibilidades de asumir una conducta acorde o no a dichos efectos.

Otra manera de evaluar los resultados de políticas multilaterales es observando el proceso desde la óptica de los propios organismos intergubernamentales. Una forma evidente de evaluar su presencia e impacto en el sistema internacional es al tenor de los efectos que produce su acción en el terreno de las relaciones interestatales, sean éstas en lo político, lo comercial, lo científico-tecnológico o en cualquier otro plano que involucre la acción específica de los Estados. 
No es equivocado suponer que la percepción que estas organizaciones tenían de su influencia ha variado en términos similares a lo descrito en el punto anterior. En un primer momento, consciente o inconscientemente, parte importante de estas organizaciones sobrevaloraron su acción y supusieron que ésta se incrementaría a futuro, acrecentando también su radio de influencia en el sistema internacional. Los efectos militares y políticos producidos por la acción pacificadora de los años cincuenta y principios de los sesenta, reforzaron esta sensación en el seno de las Naciones Unidas y se extendió hacia organismos regionales. En esta última organización, por diversas razones, los resultados fueron menos acertados (por ejemplo, en la crisis dóminicana de 1965), pero, de hecho, ello no afectó a la práctica que mantuvo en esta materia el Consejo de Seguridad, tales como la tuición militar en Chipre y en el Cercano Oriente desde mediados de los sesenta. En ese contexto, la proliferación de entidades intergubernamentales mundiales y regionales alcanza su punto cúlmine, lo cual se traduce en el acopio de una considerable cantidad de recursos que les destinan los países miembros.

La distensión, la posibilidad de solucionar concertadamente los problemas derivados de la pobreza y del subdesarrollo, ia implementación de instancias que favorecieran el poder de negociación de naciones en vías de desarrollo constituían, entre otros temas, la agenda de trabajo cotidiano en estas entidades. El desencanto de años después y la emergencia de nuevos circuitos de negociación, más pragmáticos y menos formalizados, empezaron a minar el optimismo de un principio. El costo de mantención de una burocracia internacional que desde entonces había crecido considerablemente, sumado a las dificultades de adaptación a un sistema internacional constreñido por las crisis durante el primer tercio de la década anterior, también empezaron a minar la voluntad política de muchos países hacia el multilateralismo. La inadaptación de estas organizaciones a los procesos políticos que resultan de agudas crisis recesivas, así como una peculiar impermeabilidad institucional a realidades regionales políticamente contrapuestas a las observadas en otros períodos, desvincula la percepción de dichas organizaciones de la sustentada por sus países miembros.

El pragmatismo del que está imbuido el llamado neomultilateralismo de los ochenta y de los noventa ha significado la desaparición de un estilo de diplomacia multilateral, por lo que la crisis no sólo apuntaba a meros ajustes institucionales. A la postre, la mantención 
o desaparición de un estilo diplomático depende de la voluntad política interpuesta por los Estados, voluntad a su vez supeditada a las orientaciones globales y específicas de la política exterior. La permanente evaluación de los recursos con los que esta política cuenta y las variaciones que experimenta en determinados períodos, así como una serie de indicadores directa e indirectamente relacionados con ambas, constituyen el contexto básico en el que se desenvuelven las organizaciones intergubernamentales. Su sobrevivencia institucional está ligada a su capacidad de adaptación contextual, como siempre ocurre con toda institución por lo demás.

La aplicación del esquema conductualista al estudio de las relaciones intergubernamentales en América Latina o a las que mantiene la región con otros países o grupos de países es una labor muy reciente. En la medida que ello ha implicado un reenfoque de las actividades de investigación y un énfasis en lo empírico, el respaldo institucional ha sido renuente o practicamente inexistente. No obstante, algunos avances se han logrado en este sentido y en los últimos años se observa con mayor serenidad la irrupción de metodologías heterodoxas en los centros de estudios políticos e internacionales de nuestra región.

El uso extensivo e intensivo de métodos y técnicas cuantitativas se debe, entre otras, a dos razones esenciales. Desde luego, algunos paradigmas clásicos elaborados hace cuarenta o más años han sido sobrepasados por un contexto político mundial y regional que ya no está estructurado ni funciona como entonces se prescribía. Algo adelantamos al principio de este ensayo y lo reiteramos. En segundo término, son los procedimientos estadísticos y los modelos formales (matemáticos y no-matemáticos) los que permiten ordenary sistematizar más y mejor la innumerable cantidad y calidad de variables, indicadores e índices que caracterizan a los hechos y fenómenos internacionales, además de los políticos en general. Esto no implica ni supone excluir otras metodologías y, ciertamente, los conceptos estadísticos y los modelos formales no sustituyen la capacidad interpretativa, especulativa y ensayística de la politología moderna. Es más, ha sido demostrado el aporte de modelos formales a la teorización social y política, así como están igualmente comprobados los errores a que conduce el reduccionismo cuantitivista.

En ese entendido, hemos sugerido la elaboración de un esquema formal para diagnosticar algunos conflictos intrarregionales, el cual permitiría auscultar diagnósticos y eventuales soluciones facti- 
bles. Para ello, planteamos tres hipótesis de trabajo, las que a su vez corresponden a tres escenarios posibles. En cada una se contraponen los intereses de dos partes $(X$ e $Y$ ), las cuales están política y/o estratégicamente confrontadas.

\section{HIPOTESIS DE TRABAJO I}

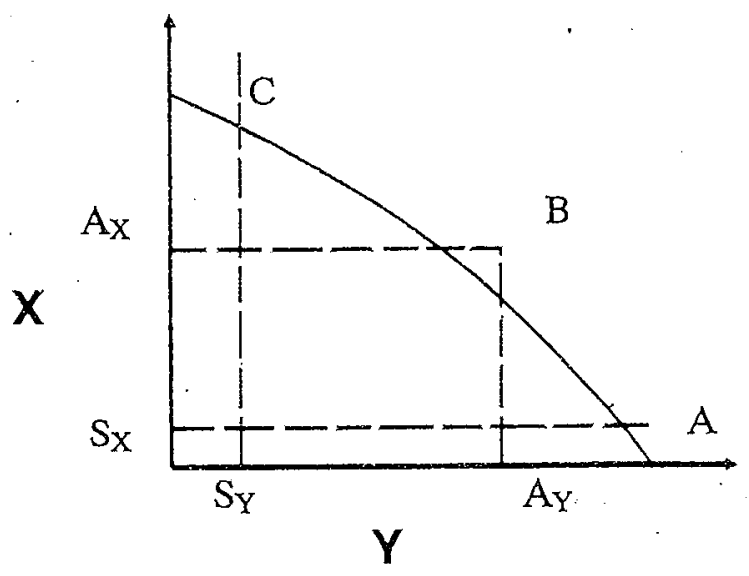

ALTA POLARIZACIÓN $\quad \Rightarrow \quad$ NULA COOPERACIÓN

-SY-C : Zona de utilidad mínima para $Y$

$-\mathrm{SX}-\mathrm{A} \quad$ : Zona de utilidad mínima para $X$

Punto B : Convergencia de intereses (fuera del sistema)

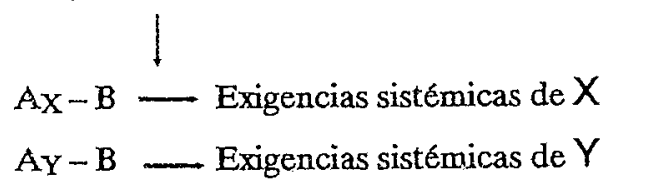


En este cuadro, las exigencias mutuas son excesivas, al menos en el contexto de un sistema con recursos limitados. Los casos en los que se traslapan geográficamente las exigencias de soberanía en territorios en disputa, constituyen un caso típico en esta hipótesis. Ello puede conducir a diferendos permanentes y la persistencia de esta situación puede agravar las relaciones y la cooperación bilateral, con todo lo que ello implica para el resto de la región.

\section{HIPOTESIS DE TRABAJO 2}

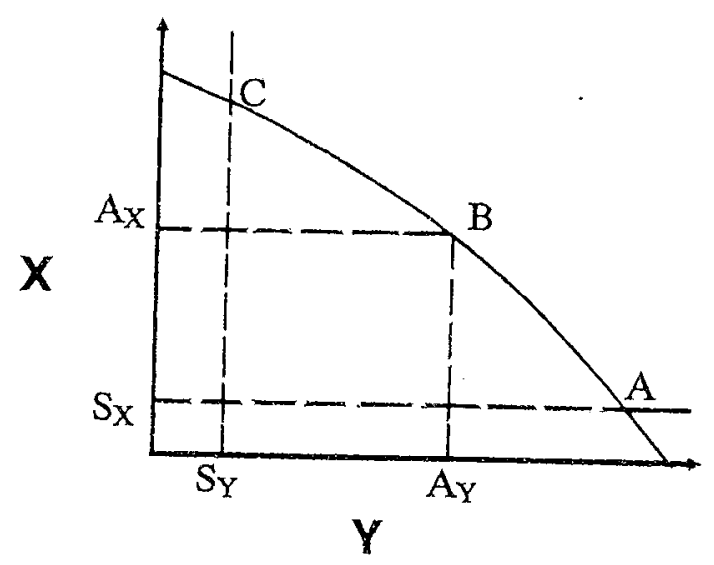

POLARIZACIONMODERADA $\Rightarrow$ MINIMA COOPERACION

$-S X-A$ : Zona de utilidad mínima para $X$

$-S Y-C \quad$ : Zona de utilidad mínima para $Y$

$A X-B \quad:$ Exigencias sistémicas de $X$

$A Y-B \quad:$ Exigencias sistémicas de $Y$

Punto B : Dentro del sistema, baja cooperación: neutralidad sistémica 
En esta hipótesis, las exigencias mutuas han disminuido, pero no lo suficiente. La curva de transformación, señala un punto (B) en el que ambos actores han cedido hasta situar sus expectativas dentro - de las posibilidades que ofrece el sistema, pero sin que ello implique una cooperación sustancial. Esta última está restringida al no-uso, por ejemplo, de la fuerza para dirimir el o los diferendos. También puede entenderse como un acuerdo tácito en orden a no exigirse mutuamente o no exigir del o al sistema más allá de un límite real y racional. Es lo que usualmente se define como crisis o conflicto limitado. Ahora bien, encontrar ese punto de cooperación mínima o neutra puede ser el producto de un largo y costoso conflicto previo.

HIPOTESIS DE TRABAJO 3

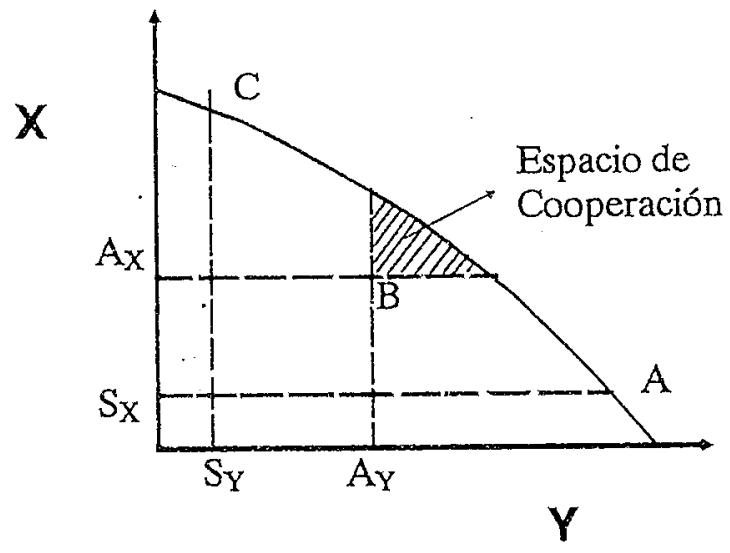

BAJA POLARIDAD $\quad \Rightarrow \quad$ ALTA COOPERACION

- SX-A : Zona de utilidad mínima para $X$

$-\mathrm{SY}-\mathrm{C} \quad$ : Zona de utilidad mínima para $Y$

$-A X-B \quad:$ Exigencias sistémicas de $X$

$-A Y-B \quad:$ Exigencias sistémicas de $Y$

Punto B : Al bajar exigencias mutuas, hay espacio para cooperar. 
Si aplicásemos -grosso modo- esta hipótesis al conflicto irako-iraní (1980-1981), por ejemplo, podría decirse que son dificultades mutuas (regionales y/o mundiales) para configurar un contexto adecuado lo que constituye un elemento explicativo de este conflicto.

En esta tercera hipótesis, se supone que los actores $\mathrm{X}$ e $\mathrm{Y}$ han disminuido sustancialmente sus exigencias mutuas y sistémicas, por lo que el vértice formado por el punto $B$ y los equidistantes en la curva de transformación han creado un espacio para cooperar. En suma, la probabilidad de establecer acuerdos o vías de avenimiento que involucren la puesta en marcha de un sistema de cooperación mutua, pasa por concesiones importantes. La curva de transformación establece un límite de recursos, la voluntad política define el tipo y grado de acercamiento con el que cada Estado se compromete a facilitar o dificultar el avenimiento bilateral y, por ende, a negociar diplomáticamente sus diferendos.

\section{REFERENCIAS BIBLIOGRAFICAS}

Adelman, Irma \& Hihn, Jairus M., "Politics in Latin America. A Catastrophe Theory Model", en: The Journal of Conflict Resolution ( ${ } C R$ ), vol. XXVI, No4, 1982, pp. 592-620.

Alexandroff, A.; Rosecrance, R.; Stein, A. "History, Quantitative Analysis, and the Balance of Power", en: The Journal of Conflict Resolution (JCR), vol. XXI, No1, 1977, pp. 35-56.

Allison, G. T. \& Halperin, M. H., "Bureaucratic Politics: A Paradigm and some Policy Implications", en: World Politics, vol. xxIv, 1972, pp. 40-79.

Axelrod, Robert (ed.), Structure of Decision The Cognitive of Political Elites, (Princeton University Press, 1976), pp. 3-75.

Bobrow, D. B. \& Stocker, R. P., "Evaluation in Foreign Policy", en: Hopman, P. T., Zinnes, D. \& Singer, J. D. (eds.), Cumulation in International Relations Research. Monograph Series in World Affairs, (Graduate School of International Studies, University of Denver, 1981), pp. 99-132.

Druckman, D.; Benton, A. A.; Ali, F.; Bagur, J. S., "Cultural Differences Bargaiging Behavior: India, Argentina, and the United 
States", en: The Journal of Conflict Resolution (JCR), vol. $\mathrm{xX}$, No3, 1976, pp. 413-452.

Eberwein, Wolf-Dieter et al., "External and Internal Conflict Behavior Among Nations; 1966-1967", en: The Joumal of Conflict Resolution (JCR), vol, XXur, $\mathrm{N}^{\circ} 4,1979$, pp. 715-742.

Goehring, D. J. \& Kahan, J. P., "The Uniform N-Person Prisoner's Dilemma Game: Construction and Test of an Index of Cooperation", en: The Journal of Conflict Resolution ( $\mathrm{JCR}$ ), vol. $\mathrm{xX}$, No1, 1976, pp. 111-128.

Heintz, Peter, "On the Change of Parameters of the International System 1870-1970. An Analysis Review of Some Recent Literature", en: The Journal of Conflict Resolution (JCR), vol. XX, $N^{\circ} 1,1976$, pp. 173-184.

Kaplowitz, Noel, "Psychopolitical Dimensions of the Middle East Conflict. Policy Implications", en: The Journal of Conflict Resolution (JCR), vol. XX, No2, 1976, pp. 279-318.

Kelman, Herbert C. (ed.), International Behavior. A Social Psychological Analysis, (New York: Holt, Rinehart and Winston, 1965).

Kramer, Marguerite \& Russett, Bruce, "Images of World Futures", en: Joumal of Peace Research (jPR), vol. 21, No4, 1984, pp. 317-336.

Korany, Baghat, Social Change, Charisma and International Behavior: Toward a Theory of Foreign Policy Making in the Third World, Sijthoff, Leiden, Collection de relations internationales No2, 1974.

Latour, S.; Houlden, P.; Walker, L.; Thibault, J., "Some Determinants of Preference for Modes of Conflict Resolution", en: The Journal of Conflict Resolution (ICR), vol. XX, No2, 1976, pp. 319-356.

Levi, A. M. \& Benjamin, A., "Focus and Flexibility in a Model of Conflict Resolution", en: The Journal of Conflict Resolution (JCR), vol. XX, No2, 1977, pp. 405-426.

Ostrom, Charles W. Jr., "Evaluating Alternative Foreign Policy Decision-Making Models: An Empirical Test between an Arms Race Model and an Organizational Politics Models", en: The Journal of Conflict Resolution (JCR), vol. XXI, N22, 1977, pp. 235-266. 
Schleicher, Charles P., International Behavior. Analysis and Operations, (Ohio: Charles E. Merril Publications Co., 1973), pp. 49-68, 69-84.

Schroeder, Paul W., "Quantitative Studies on the Balance of Power: An Historian's Reaction", en: The Journal of Conflict Resolution (JCR), vol. XXr, $\mathbb{N}^{\circ} 1,1977$, pp. 3-22.

Smith, B., "A Map for the Analysis of Personality and Politics", en: The Joumal of Social Issues, vol. XxIV, 1968, pp. 15-28.

Sylvan, Donald A., "Planning Foreign Policy Systematically. Mathematical Foreign Policy Planning", en: The Joumal of Conflict Resolution (JCR), vol. XxIII, No1, 1979, pp. 139-173.

Thompson, W. R. \& Modelski, G., "Global Conflict Intensity and Great Power Sommitry Behavior", en: The Journal of Conflict Resolution (JCR), vol. XXI, N'2, 1977, pp. 339-376.

Väyrynen, Raimo, "Regional Conflicts Formations: An Intractable Problem of International Relations", en: Journal of Peace Research (JPR), vol. 21, No4, 1984, pp. 337-359.

The Journal of Conflict Resolution, vol. XxI, N4, 1977. (Todo el número)

Young, Jerald W., "Behavioral and Accepted Differences between Structurally Equivalent Two-Person Games: A Rich-Poor Context Comparison", The Journal of Conflict Resolution (JCR), vol. XXI,N2, 1977, pp. 299-322. 